\title{
ESTUDO EXPERIMENTAL DA RESISTÊNCIA DAS OSTEOSSÍNTESES COM PLACAS E PARAFUSOS NA FIXAÇÃO ANTERIOR DA COLUNA CERVICAL
}

\section{RESISTANCE OF OSTEOSYNTHESES WITH PLATES AND SCREWS IN ANTERIOR CERVICAL SPINE FIXATION: AN EXPERIMENTAL STUDY}

José Sérgio Franco ${ }^{1}$ Itibagi da Rocha Machado², Reginaldo Perillo de Olinelra ${ }^{3}$, Alexandre Fogaça Cristantél, Tomas Puga Leivas 6 , Raphael Martus Marcon ${ }^{5}$, Almir Fernando Barbarini ${ }^{5}$, Tarcísio Eloy Pessoa de Barros Fllho

\section{RESUMO}

Com o objetivo de verificar a resistência das osteossínteses com placas e parafusos, por via anterior, o autor realizou estudo experimental em segmentos da coluna cervical (C3-C7) de cadáveres frescos, comparando três diferentes tipos de placas. Utilizou placa tipo $\mathrm{H}$ de Orozco (4 peças), placa convencional de 1/3 de tubo (4 peças), placa de Mendonça (5 peças) e o Grupo Controle sem osteossíntese (5 peças). Em todas as 18 peças foi realizado corpectomia central sem destruição das paredes laterais do corpo vertebral. As peças foram testadas em máquina de compressão axial, com registrador gráfico mecânico, sendo aplicadas cargas lentas e progressivas. Os resultados mostraram, em relação à falha inicial, que as estabilizações das osteossínteses são semelhantes entre elas, mas inferiores ao Grupo Controle. Estatisticamente não é significativa a diferença de estabilidade entre o Grupo com a placa $\mathrm{H}$ e o Grupo Controle, entretanto existe diferença entre este e os grupos com as placas de 1/3 de tubo e de Mendonça. Em relação à resistência máxima, não houve diferença significativa na comparação entre as osteossínteses e entre estas o Grupo Controle. Com base nos resultados, o autor conclui que a osteossíntese com placa $\mathrm{H}$ confere maior estabilidade quando comparada às outras osteossínteses, porém as placas e parafusos utilizados diminuíram a estabilidade, determinando que a falha inicial ocorresse precocemente, quando comparada ao Grupo Controle.

Descritores: Traumatismos da coluna; Fixação interna de frauturas; Parafusos ósseos; Placas ósseas; Cadáver.

Citação: Franco JS, Oliveira RP, Cristante AF, Leivas TP, Marcon RM, Barbarini AF et al. Estudo experimental da resistência das osteossínteses com placas e parafusos na fixação anterior da coluna cervical. Acta Ortop Bras. [periódico na Internet]. 2007; 15(4):191-196. Disponível em URL: http://www.scielo.br/aob.

\section{INTRODUÇÃO}

As lesões traumáticas da coluna cervical, de forma geral, resultam para a sociedade, em graves problemas de natureza sócio-econômica. Por exemplo: a incidência da lesão medular nos Estados Unidos chega a 50 casos por milhão de habitantes por ano, o que significa cerca de 10.000 casos novos a cada ano. O custo aproximado para a sociedade, a cada nova lesão cervical com déficit neurológico, foi estimado pela American Spinal Injury Association em U\$ 400.000 dólares. O custo do tratamento médico destes pacientes a curto prazo alcançou, no ano de 1980, nos Estados Unidos, cifras aproximadas de U\$\$ 1.5 bilhões de dólares, enquanto o custo total para a sociedade e para todos os lesados medulares

\section{SUMMARY}

In order to evaluate the stability of the anterior fixation with plate and screws, the author conducted an experimental study in segments of the cervical spine (C3-C7) in fresh human cadavers, comparing 3 different types of plates. He used a H-type Orozco's plate (4 specimens); a conventional plate of $1 / 3$ tubular (4 specimens); plates described by Mendonça (5 specimens); and the control group without fixation (5 specimens). In all specimens a Corpectomy was used and the lateral walls of the vertebral bodies were left intact. The specimens were tested in axial compression machine, with slow and progressive loading, using mechanical graphic recorder. Results have shown that, concerning initial failure, stabilization of the fixations is similar among groups but inferior to the control group. Statistically, the difference was significant between the control group and the 1/3 tubular and Mendonça plates, but it was similar to the $\mathrm{H}$ plate. Concerning maximum resistance, no significant differences were observed in comparing fixations to the control group. Based on the results, the author concludes that fixation with the $\mathrm{H}$ plate offers better stability when compared to other fixations and that the plates and screws used in the study decreased resistance when compared to the control group.

Keywords: Spinal injuries; Fracture fixation, internal; Bone plates, Bone screws; Cadaver.

Citation: Franco JS, Oliveira RP, Cristante AF, Leivas TP, Marcon RM, Barbarini AF et al. Resistance of osteosyntheses with plates and screws in anterior cervical spine fixation: an experimental study. Acta Ortop Bras. [serial on the Internet]. 2007; 15(4): 191-196. Available from URL: http://www.scielo.br/aob.

alcançou cifras de U\$\$ 4 bilhões de dólares ${ }^{(1)}$. Embora estes valores indiquem um problema relevante, a tragédia de uma lesão medular só é totalmente compreendida quando se avaliam de maneira mais ampla as suas conseqüências na vida dos pacientes, em particular os que apresentam paraplegia ou tetraplegia, estando a maioria deles na terceira década de vida.

As lesões da medula cervical são incapacitantes, na maioria das vezes, de forma permanente, visto que as técnicas modernas de tratamento ainda pouco conseguem para a restauração das funções neurológicas.

Fatores anatômicos e biomecânicos fazem da coluna cervical uma região crítica no trauma vertebral, a estes se associam falhas de

Trabalho realizado no Instituto de Ortopedia e Traumatologia. Laboratório de Investigação Médica do IOT/HC/FMUSP (LIM-41)

Endereço para Correspondência: Rua José Maria Lisboa, 285 apto 41 - Jardim Paulista - São Paulo - SP - Brasil - CEP 01423001 - almirbarbarini@terra.com.br

1. Professor Adjunto da Universidade Federal do Rio de Janeiro (UFRJ)

2. Doutor da Faculdade de Medicina de Jundiai

3. Médico Chefe do Grupo Coluna do Instituto de Ortopedia e Traumatologia da Faculdade de Medicina da Universidade de São Paulo, São Paulo - Brasil (IOT/HC/FMUSP)

4. Médico Assistente do Grupo Coluna do IOT/HC/FMUSP

5. Médico Colaborador do Grupo de Coluna do IOT/HC/FMUSP

6. Engenheiro Chefe do Laboratório de Biomecânica - LIM-41 do IOT/HC/FMUSP

7. Professor Titular do Departamento de Ortopedia e Traumatologia da Faculdade de Medicina da Universidade de São Paulo, São Paulo - Brasil

Trabalho recebido em 11/10/06 aprovado em 10/11/06 
primeiro atendimento, dificuldades de diagnóstico e de tratamento precoce, além da possibilidade de lesões associadas.

A literatura disponível mostra um evidente evolução na observação de traumas da coluna vertebral, como o estudo da unidade vertebral, isto é, o segmento formado por duas vértebras intactas, disco intervertebral e ligamentos; também são estudadas as unidade funcionais representativas da coluna, simulando lesões cervicais em cadáveres ${ }^{(2,3)}$

A biomecânica da coluna cervical ainda não é bem conhecida devido às peculiaridades das várias estruturas que a compõem, e também por sua complexidade fisiológica e anatômica(4)

A biomecânica reúne conhecimentos acumulados e disponíveis nos diversos ramos da tecnologia, utilizando-os em conjunto nas diversas áreas da Medicina e da Biologia. Uma grande contribuição é fornecida pelo desenvolvimento de modelos experimentais que simulam movimentos e traumas cervicais. A insuficiente interpretação de resultados experimentais decorre, às vezes, da ausência de ação estabilizadora dos músculos, influências estas que não podem ser totalmente reproduzidas e analisadas. O mecanismo do trauma, por envolver forças combinadas de intensidade, direção e velocidade variáveis, também torna difícil sua reprodução em condições experimentais. A pesar destas e de outras limitações, acredita-se que estes estudos permitam reproduzir comparações de vários tipos de fixação interna e possíveis interações entre a coluna vertebral do espécime estudado e o implante.

Em 1955, a fusão cervical anterior tornou-se um procedimento universalmente aceito na artrodese, em nível único, nas doenças degenerativas cervicais ${ }^{(5)}$. Infelizmente, não têm sido obtidos os mesmos resultados nas fraturas, ressecções tumorais e deformidades em cifose, onde têm sido observadas inaceitáveis taxas de deformidades progressivas, instabilidade e extrusão do enxerto(5). Desde 1964 os sistemas de fixação - placas convencionais ${ }^{(6,7)}$ e tipo H de Orozco ${ }^{(8)}$ - têm sido utilizados, embora sem aceitação universal, devido aos riscos de lesão medular, radicular e vascular durante a sua colocação. Lesões do esôfago e de outras estruturas vizinhas secundárias à migração tardia de parafusos, de placa ou enxerto ósseo, também têm sido descritas ${ }^{(9)}$.

No sistema tradicional placa-parafuso não bloqueado, os parafusos terão maior fixação ao osso se ultrapassarem a parede posterior do corpo vertebral e forem colocados obliquamente, em direção ao disco intervertebral.

No sistema placa-parafuso bloqueado, descrito em $1986^{(10)}$, os parafusos não perfuram a parede posterior do corpo vertebral, o passo de rosca é menor e eles são colocados em direção convergente, proporcionando maior fixação ao osso.

Inúmeros tipos de instrumentação também foram desenvolvidos para a fixação da coluna cervical através de acesso posterior ${ }^{(7,11-16)}$ e estariam indicados, principalmente, nas luxações irredutíveis por métodos conservadores.

Nos traumatismos raquimedulares cervicais devido a fratura por explosão do corpo vertebral, com fragmentos ósseos dentro do canal medular, está indicada a descompressão anterior, enquanto persiste a discussão do acesso cirúrgico anterior, posterior ou associado para a estabilização de outras lesões traumáticas da coluna cervical.

Nas fraturas, tumores ou deformidades cifóticas, eventualmente, está indicado um segundo procedimento (via posterior) para maior estabilização. Para evitar um segundo acesso cirúrgico após a "corpectomia*" surgiu o conceito de enxertia óssea anterior associada à fixação com placas e parafusos ${ }^{(6-8,10,17-19)}$. Esta fixação pelo mesmo acesso anterior da corpectomia pode ser feita com placas e parafusos, tais como: placa tipo $\mathrm{H}^{(8)}$, tipo trapezoidal(18), retas convencionais de $1 / 3$ de tubo ${ }^{(6,7)}$ e retas com ganchos proximal e distal(20).

Em razão da existência de inúmeros questionamentos quanto à utilização de placas e parafusos na coluna cervical, tais como sustentação, estabilização e absorção de tensões exercidas sobre o conjunto vertebral, comparamos diferentes tipos de placas, através de determinado modelo experimental, já utilizado anteriormente por outros autores ${ }^{(21-23)}$. Os conjuntos vertebrais cervicais foram submetidos a testes de compressão axial, após a corpectomia da vértebra intermediária (C5) e fixação dos parafusos nas vértebras cervicais adjacentes (C4-C6). A falha inicial e a resistência máxima destes conjuntos vertebrais fixados e do grupo controle, oram então identificados.

\section{MATERIAL E MÉTODO}

Foram utilizadas 19 colunas vertebrais cervicais humanas, retiradas de cadáveres do sexo masculino, com idade variável de 25 a 40 anos, com diagnóstico anátomo-patológico não relacionado com doença que envolvesse comprometimento ósseo e cujo óbito ocorrera menos de 24 horas antes da retirada do material.

O material estudado, durante os anos de 1990 e 1991, pertencia ao Serviço de Verificação de Óbitos da Faculdade de Medicina da Universidade de São Paulo.

A preparação do material foi realizada com os cadáveres em decúbito ventral, a cabeça colocada além da borda da mesa anatômica, de tal maneira que o pescoço permaneceu em hiperflexão pela ação da gravidade. A seguir, por meio de incisão cervical longitudinal posterior, a peça foi retirada em bloco único, desde a primeira vértebra cervical até a primeira vértebra torácica, mantidos cápsulas, disco e ligamentos. Neste segmento da coluna vertebral, procedeu-se à separação do conjunto C3, C4, C5, C6 e C7 (da terceira à sétima vértebra cervical), identificado a partir do axis (C2).

Cada um dos conjuntos vertebrais foi colocado em saco de polietileno, com a máxima retirada de ar do seu interior, fechado hermeticamente e mantido em congelador à temperatura de 20 graus Celsius negativos. Na preparação para as simulações, cada coluna foi retirada do congelador e colocada à temperatura e à umidade ambiente numa bandeja plástica, com solução de cloreto de sódio a 0,9\%, onde as peças ficaram em banho isotônico, no mínimo por duas horas. A seguir as peças foram dissecadas, retirando-se as partes moles de C3, C4, C5, C6 e C7, exceto a cápsula articular, os ligamentos e os disco intervertebrais.

As peças a serem testadas foram divididas em quatro grupos, com o objetivo de estudas três diferentes tipos de osteossíntese e mais o Grupo Controle. Todos foram submetidos à corpectomia central, isto é, ressecção de aproximadamente $1 / 3$ do diâmetro transverso do corpo vertebral, incluindo as paredes anterior e posterior, da quinta vértebra cervical (C5) (Figura 1), associada à retirada dos discos intervertebrais e dos ligamentos longitudinais anterior e posterior de C4-C5 e C5-C6. Tal procedimento imitou, dentro do possível, o ato operatório. A parte central do corpo vertebral e as partes moles adjacentes foram retiradas, por partes, com auxílio de pinças cirúrgicas. O espaço vazio correspondente à corpectomia é limitado superiormente pela face inferior do corpo de C4, inferiormente pela face superior do corpo de C6 e lateralmente pelas paredes laterais do corpo vertebral de C5. Após este procedimento, foi realizada a fixação interna utilizando três diferentes tipos de placas e fixação com parafusos tipo cortical, de diâmetro de $3.5 \mathrm{~mm}$ e comprimento $16 \mathrm{~mm}$, sobre a face anterior do corpo vertebral de $\mathrm{C} 4$ e C6.

A placa tipo $\mathrm{H}$ - descrita por Orozco e Llovettapies ${ }^{(8)}$, tem uma concepção peculiar, pois é fixada por dois parafusos corticais proximais e dois distais, também de diâmetro de $3.5 \mathrm{~mm}$ e comprimento $16 \mathrm{~mm}$, tendo um efeito anti-rotatório (Figura 2). A placa de $1 / 3$ de tubo $3.5 \mathrm{~mm}-\mathrm{AO}^{(6,7)}$ é uma placa convencional estreita e reta, a ser fixada por um parafuso tipo cortical - de diâmetro de $3.5 \mathrm{~mm}$ e com $16 \mathrm{~mm}$ de comprimento - proximal e outro distal. A placa Mendonça ${ }^{(20)}$, desenvolvida na Faculdade de Medicina da Universidade de São Paulo, apresenta também um conceito

*Nota do autor: A palavra corpectomia não existe na língua portuguesa. Entretanto, foi usada no presente trabalho por não ter sido encontrada palavra equivalente em nossa língua para o termo inglês Corpectomy. Foi também citada por BARROS FILHO³, em 1990 - USP. 
diferente das duas placas anteriores, por apresentar duas garras proximais e duas garras distais, além da fixação com um parafuso tipo cortical de comprimento de $16 \mathrm{~mm}$ e de diâmetro $3.5 \mathrm{~mm}$ - proximal e outro distal (Figura 3).
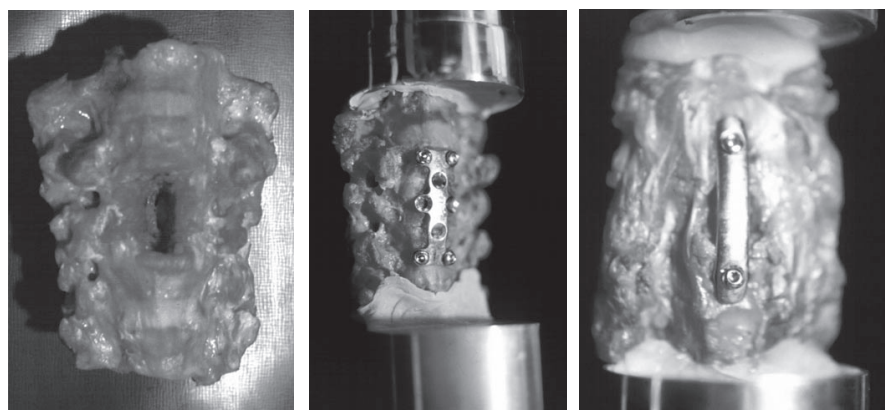

Figura 1 - Conjunto Figura 2 - Conjunto Figura 3-Conjunto cervical cervical (C3 - C7) cervical (C3 - C7) (C3 - C7) mostrando a mostrando acorpectomia mostrando a fixação fixação com placa em C5. Neste estudo com placa tipo $\mathrm{H}$ em Mendonça em C4 e C6 foram utilizados três C4 e C6 diferentes tipos de placas para a fixação anterior da coluna cervical que representam três diferentes conceitos de fixação.

Não foi utilizado enxerto ósseo ou cimento acrílico para preencher o espaço correspondente à corpectomia, para que a placa e as paredes laterais do corpo vertebral e as estruturas discoligamentares suportassem o efeito de compressão axial do modelo experimental.

Além dos três grupos de osteossíntese, um outro grupo com cinco peças foi utilizado como Controle, no qual era realizada apenas a corpectomia central em C5, sem fixação interna.

A título de comparação, mais uma peça foi testada, na qual foram seccionadas as paredes laterais do corpo vertebral de C5 após a corpectomia centreal, sem osteossíntese, funcionando como Teste Piloto.

Os quatro grupos de testes e seus respectivos tipos de osteossínteses e o número de casos estão distribuídos na (Tabela 1).

\begin{tabular}{c|c|c}
\hline № DA PEÇA & GRUPOS & TIPO DE PLACAS \\
\hline 5 & G I & Controle \\
\hline 4 & G II & H Orozco \\
\hline 4 & G III & $1 / 3$ tubo \\
\hline 5 & G IV & Mendonça \\
\hline
\end{tabular}

Fonte: FM - USP

Tabela 1 - Número de peças por tipo de placa testada.

Para execução dos testes, realizou-se, para cada peça, um ensaio de compressão axial, com cargas lentas e progressivas, empregando-se uma máquina universal de ensaio mecânicos Kratos 5062, dotada de célula de carga eletrônica CCT-10.000 Kfg. Todos os experimentos foram acompanhados por registrador gráfico mecânico SERVOGOR 790 BBC Goerz Metravatt, onde a deformação em abcissar foi ajustada em milímetro (mm), e a carga, em ordenadas em quilograma-força (Kgf). Nos ensaios, adotamos a escala de $2.500 \mathrm{Kgf}$ e velocidade constante do travessão móvel de $20 \mathrm{~mm} / \mathrm{min}$, com bloqueio de experimento, após ser observado nos gráficos o segundo pico de carga (Resistência Máxima).

Foram registrados para análise, os valores da falha inicial e da resistência máxima. A falha inicial foi considerada no primeiro sinal de diminuição da resistência do conjunto pesquisado, que foi registrado nos gráficos como primeiro pico de carga. A seguir prosseguiu-se com o ensaio até a ocorrência de falha completa de resistência do conjunto - resistência máxima -, que foi registrada nos gráficos, como segundo pico de carga, em quilograma-força (Kgf).

Os ensaios mecânicos foram efetuados no Laboratório de Investigação Médica, LIM - 41, do Departamento de Ortopedia e Traumatologia da Faculdade de Medicina da Universidade de São Paulo.

Foram realizados testes preliminares em um conjunto cervical extra, igual ao do trabalho, com finalidade de verificar o método e ajustar os equipamentos envolvidos.

Para garantirmos os níveis de precisão nos ensaios de compressão, empregamos cargas axiais uniformes, através de superfícies planas e paralelas, entre as placas da máquina e os corpos de prova. Para obter estas condições e a homogeneização da carga, sem danificar as peças, recobrimos as superfícies de interface - plataforma - osso - com cimento acrílico, preenchendo as irregularidades e moldando mais adequadamente as superfícies ao teste (Figura 2).

Para o apoio das peças, utilizou-se um dispositivo especial de ajuste manual, fixado à base da máquina, composto por dois tubos rosqueados. Para regulagem de aproximação das plataformas, obteve-se a elevação girando-se os tubos em sentido anti-horário. O tubo interno recebe o encaixe de um cilindro em sua porção superior e, na superfície deste, sobrepõe-se um disco tipo bandeja para limitar os movimentos da peça, servindo, também, para limpeza após o teste.

Foram realizados estudos radiográficos dos segmentos cervicais (C3 a C7) na incidências ântero-posterior (AP) e perfil, antes e após o ensaio mecânico.

O objetivo específico desta avaliação foi constatar a posição dos parafusos - inclinação em relação aos discos intervertebrais e a não penetração na parede posterior do corpo vertebral - e, conseqüentemente, padronizar os efeitos do conjunto das placas e parafusos sobre os segmentos vertebrais estabilizados.

Para as análises estatísticas dos resultados foram empregados os testes de Mann-Whitney e de análise de variância, tendo sido estabelecido nível de significância de $5 \%(p<0,05)$ para ambos os testes.

\section{RESULTADOS}

Os resultados no estudo da falha inicial na comparação do Grupo Controle demonstrou que as médias dos grupos com fixação foram menores que as do Grupo Controle, em ordem crescente (Grupos IV, III e II - Mendonça, 1/3 de tubo AO e H). Não houve diferença entre o Grupo Controle e Grupo II (placas tipo H). Como foi significante a diferença entre os dois grupos, Mendonça $(p=0,016)$ e 1/3 de tubo AO $(p=0,032)$ e o Grupo Controle, pode-se depreender que estas placas produzem falha inicial mais precocemente.

Em comparação a falha inicial dos grupos entre si demonstrou diferença significativa entre as placas em H Orozco e as placas de $1 / 3$ de tubo $A O(p=0,032)$, assim como houve diferença significativa entre as placas em $\mathrm{H}$ Orozco e as placas de Mendonça $(p=0,032)$. (Tabela 2)

O Grupo Controle, em relação à falha inicial, apresentou valores superiores ao teste piloto (um caso). Esta maior resistência se deve à integridade das paredes laterais do corpo vertebral. $\mathrm{Na}$ ausência das paredes íntegras a resistência foi muito menos, aproximando-se dos resultados dos testes com a placa de Mendonça, teoricamente a mais resistente e, portanto, a que conferiu o maior componente de rotação nos testes de compressão axial.

Na comparação entre o Grupo das placas de 1/3 de tubo AO, placas de Mendonça e placas $\mathrm{H}$ com o Grupo Controle, é demonstrado que todas as placas apresentam resistência máxima inferior na média ao Grupo Controle, embora esta diferença não seja significante pelo teste $U$ de Mann-Whitney, sendo em ordem crescente, as placas de Mendonça a menor média, as placas 1/3 de tubo AO a média intermediária, e as placas $\mathrm{H}$ a maior média das três.

Na avaliação da resistência máxima a comparação entre os grupos demonstrou não houve diferença estatística e ocorreu, em ordem crescente, as placas de Mendonça com a menor média, as placas 
1/3 de tubo AO com média intermediária, e as placas H com a maior média. (Tabela 3)

Comparando-se as médias da resistência máxima dos quatro grupos com as peças do experimento piloto, constatou-se que a resistência máxima desta peça, isto é, com ruptura das paredes laterais do corpo vertebral que foi submetido a corpectomia central (C5), era discretamente superior à média do Grupo Mendonça e inferior ao da placa 1/3 de tubo. Também o Grupo de Placa H Orozco mostrou a média de resistência máxima maior do que a do caso piloto. O Grupo Controle foi o que mostrou maior resistência ao se comparar com o caso piloto, certamente graças à integridade das paredes laterais do corpo vertebral.

\section{DISCUSSÃO}

As informações obtidas do comportamento mecânico das estruturas vertebrais são baseadas em pesquisas biomecânicas(24), que utilizam conceitos, princípios, termos, metodologias, cálculos, além de modelos matemáticos para interpretação e análise da anatomia e da fisiologia. A formulação de novos modelos deve ser contínua, sendo necessário contudo que haja uma progressiva melhora na precisão dos dados decorrentes de sua utilização e que estes modelos sejam cada vez mais econômicos, produzindo resultados estatísticos importantes com custos menores ${ }^{(5,25,26)}$.

Durante o desenvolvimento de uma pesquisa biomecânica, numa área fronteiriça é necessário que o estudo seja multidisciplinar, contando-se com a colaboração de médicos, bioengenheiros, estatísticos, engenheiros e técnicos em computação( ${ }^{(5)}$. Neste sentido, para que todos possam falar uma linguagem comum, facilitando a comunicação entre os integrantes desta equipe multidisciplinar, faz-se necessário conhecer termos e conceitos de biomecânica(27). Além disto, deve-se ressaltar a importância do laboratório de pesquisa estar localizado dentro da instituição médica, por isto propicia a operacionalização adequada das linhas de pesquisa,

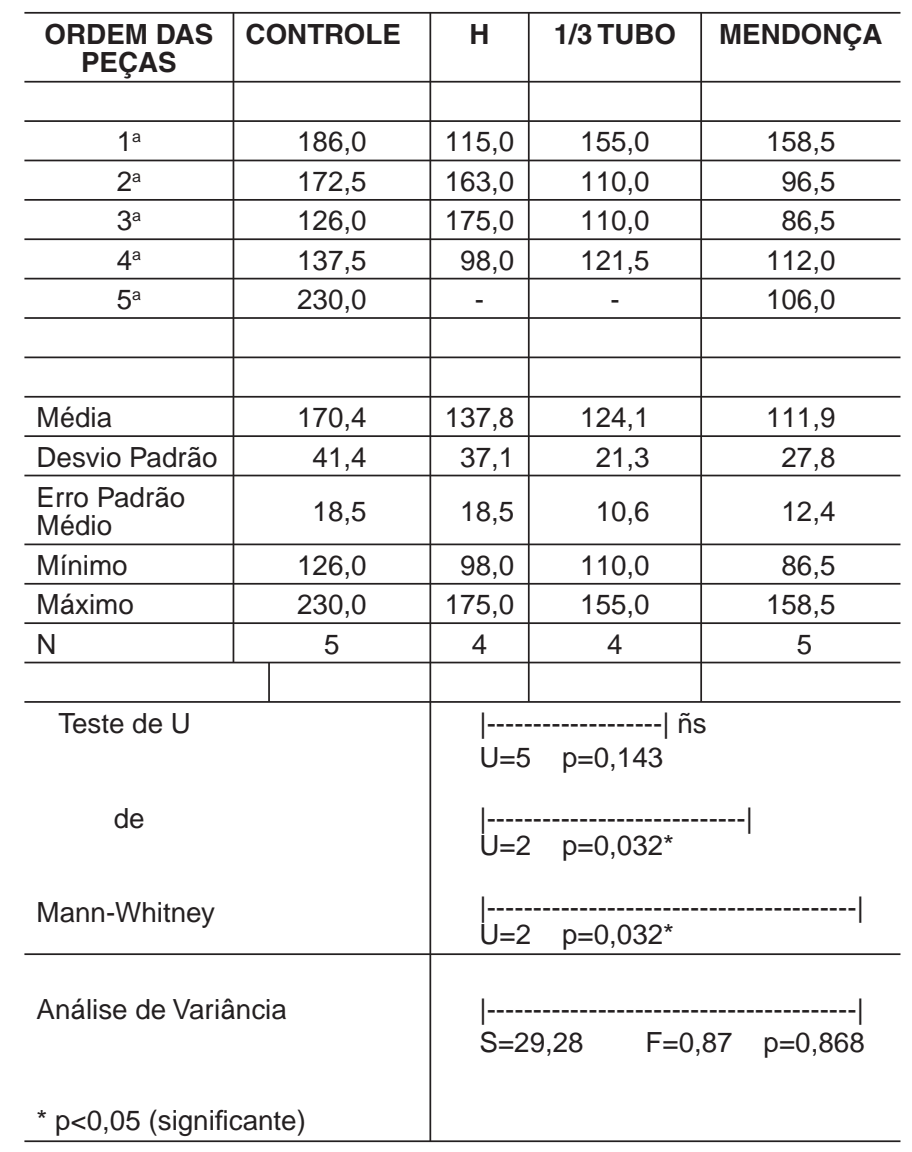

Tabela 2 - Falha inicial (Kfg) de todos os grupos. racionalizando os recursos e permitindo a dinamização constante dos conhecimentos e maior repercussão destes.

O modelo experimental utilizado na presente pesquisa foi desenvolvido em nosso meio e utilizado por outros autores ${ }^{(21-23)}$, eqüivalendo, in vivo, ao pós-operatório imediato de uma fixação interna por acesso anterior. Trata-se de modelo adequado ao estudo proposto, embora se saiba que a tendência é para que se realizem estudos cíclicos, que são os que se aproximam mais adequadamente dos movimentos diários de um indivíduo(24,28,29) É sabido que in vivo, as falhas ocorrem em decorrência dos movimentos repetitivos, e portanto, esta seria a situação ideal para a realização dos testes (24). Apesar das limitações, o modelo utilizado permitiu o estudo da falha inicial e da resistência máxima através da simulação de uma lesão por flexão-compressão, segundo classificação de Allen et al. $^{(30)}$, Denis ${ }^{(31)}$, Eurel e Kazarian ${ }^{(32)}$, Holdsworth ${ }^{(33)}$, Panjabi et al. ${ }^{(34)}$. Uma das limitações deste modelo seria a aplicação de carga lenta e progressiva e não a utilização de carga de aplicação rápida, imitando o impacto que ocorre nas lesões da coluna cervical. As forças são aplicadas em baixa velocidade para facilitar a análise, pois são obtidos resultados similares em experimentos de alta velocidade (impacto), segundo Roaf(2).

As máquinas universais de testes são utilizadas para análise de diversas propriedades físicas de materiais e também vêm sendo utilizadas para investigar e testar a resistência de tecidos biológicos, segundo Davis(35) e Shimano et al.(25). No presente trabalho a máquina utilizada (Kratos) demonstrou ser eficaz na simulação de compressão durante os ensaios programados, confirmando experiências anteriores de Machado(21), Puertas( ${ }^{(22)}$ e Rossi et al. (23). Também resultou adequada a fixação das peças à máquina por meio do uso de cimento acrílico. Isto evitou movimentos indesejáveis nas extremidades dos conjuntos vertebrais, fato já comprovado anteriormente por Miller et al. ${ }^{(36)}$ e Rossi et al. ${ }^{(23)}$.

A prática do congelamento das peças a $20^{\circ}$ Celsius negativos

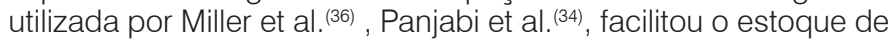

\begin{tabular}{|c|c|c|c|c|}
\hline $\begin{array}{l}\text { ORDEM DAS } \\
\text { PEÇAS }\end{array}$ & CONTROLE & $\mathbf{H}$ & 1/3 TUBO & MENDONÇA \\
\hline $1^{a}$ & 238,5 & 277,5 & 300,5 & 281,5 \\
\hline $2^{a}$ & 535,0 & 236,5 & 326,5 & 185,0 \\
\hline $3^{a}$ & 250,0 & 312,5 & 223,0 & 254,5 \\
\hline $4^{a}$ & 168,5 & 372,0 & 227,5 & 294,0 \\
\hline $5^{a}$ & 381,5 & - & - & 204,0 \\
\hline Média & 314,7 & 299,6 & 269,4 & 243,8 \\
\hline Desvio Padrão & 145,2 & 57,4 & 52,1 & 47,7 \\
\hline $\begin{array}{l}\text { Erro Padrão } \\
\text { Médio }\end{array}$ & 64,9 & 28,7 & 26,0 & 21,3 \\
\hline Mínimo & 168,5 & 236,5 & 223,0 & 185,0 \\
\hline Máximo & 535,0 & 372,0 & 326,5 & 294,0 \\
\hline $\mathrm{N}$ & 5 & 4 & 4 & 5 \\
\hline \multicolumn{2}{|l|}{ Teste de U } & \multicolumn{3}{|c|}{$U=9 \quad p=0,452 \tilde{n} s$} \\
\hline \multicolumn{2}{|l|}{ de } & \multicolumn{3}{|c|}{ U } \\
\hline \multicolumn{2}{|l|}{ Mann-Whitney } & \multicolumn{3}{|c|}{$\mathrm{U}=11 \quad \mathrm{p}=0,650$ ñs } \\
\hline \multirow{2}{*}{\multicolumn{2}{|c|}{ Análise de Variância }} & \multicolumn{3}{|c|}{ 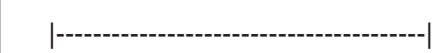 } \\
\hline & & $S=5$ & $2,07 \quad F=1,28$ & $\mathrm{p}=0,321$ ñs \\
\hline
\end{tabular}

Tabela 3 - Resistência máxima (Kgf) de todos os Grupos. 
peças e sua utilização dentro das disponibilidades da equipe. 0 descongelamento à temperatura ambiente funcionou adequadamente, sem que as propriedades biomecânicas das peças fossem alteradas de modo significativo. Entretanto, Sedlin e Hirsch ${ }^{(37)}$ e Tkaczuk ${ }^{(38)}$ consideram que o congelamento e descongelamento das peças provocam alteração na estrutura e nas propriedades biomecânicas destas. Este aspecto, entretanto, não pareceu relevante no presente estudo.

As peças estudadas foram selecionadas dentro de uma faixa etária compreendida entre 25 e 40 anos, com objetivo de obter resultados homogêneos em relação à resistência osteo-ligamentar. Evitou-se usar peças de indivíduos idosos, pois segundo Atinkson ${ }^{(39)}$, há uma progressiva fragilidade óssea após 50 anos, o que determina uma substancial redução de resistência mecânica. Vale ressaltar também que a extensibilidade dos ligamentos diminui com a idade, segundo Traczuk ${ }^{(38)}$.

A estabilidade conferida pelas estruturas musculares, ligamentares, capsulares e discais in vivo é reconhecida por Beatson ${ }^{(40)}$, Gosh ${ }^{(41)}$, Johnson et al. ${ }^{\left({ }^{(4)}\right)}$, Nova Monteiro e Giesta(43), Panjabi et al. ${ }^{(4)}$, como de grande importância. No modelo pesquisado as estruturas cápsulo-ligamentares e discais foram preservadas. Os músculos cervicais não foram levados em consideração pela inerente dificuldade em testá-los, apesar de ser reconhecida a importância desta ação muscular na estabilidade em indivíduo acordado, como citado por Fielding et al. ${ }^{(44)}$, Gosh et al. ${ }^{(41)}$, Nolan e Sherk(45), Yoganandam et al. ${ }^{\left({ }^{(3)}\right.}$. Em relação a esta ação Halliday et al. ${ }^{(46)}$, White 3 rd et al. ${ }^{(47)}$ afirmam exatamente o contrário.

Em estudo experimental de trauma em flexão do segmento médio e inferior da coluna cervical, Machado(21) observou que o ligamento longitudinal anterior não foi lesado em nenhuma das 40 peças testadas, o que coincide com a observação de vários autores: Davis ${ }^{(35)}$, Holdsworth ${ }^{(33)}$, Johnson et al. ${ }^{(42)}$, Panjabi et al. ${ }^{(48)}$, Tkaczuk ${ }^{(38)}$, White 3rd et al. ${ }^{(47)}$. É evidente que a lesão deste ligamento pode ocorrer no trauma em hiperextensão, permanecendo íntegro, na maioria das vezes, o ligamento longitudinal posterior. Nas lesões instáveis em flexão todo complexo ligamentar posterior está danificado, restando íntegro, na maioria das vezes, o ligamento longitudinal anterior, segundo Danis ${ }^{(31)}$ e White 3rd et al. ${ }^{(47)}$. Quando indicada a descompressão anterior (corpectomia) parece um contra-senso a ressecção, a este nível, do ligamento longitudinal anterior, o que acarretaria instabilidade. Nestes casos, a osteossíntese conferiria estabilidade suficiente ao conjunto vertebral fixado, após a corpectomia.

Em relação ao complexo cápsulo-ligamentar dos conjuntos vertebrais, foram observadas poucas alterações durante a realização dos testes em flexão-compressão. A rigor, as rupturas ocorreram basicamente no nível da estrutura óssea, dentro do limite das cargas imprimidas para testar a resistência máxima, quando então o teste era interrompido.

Vários trabalhos mostram que no traumatismo raquimedular as vértebras mais comprometidas na coluna cervical são C5 e C6, sendo mais freqüente a vértebra $C 5^{(21,49,50,51)}$. Este foi um dos motivos para a escolha do nível da corpectomia e, além disto, o modelo experimental revelou-se mais adequado, pois restavam duas vértebras acima e duas abaixo do nível da corpectomia central (C5), de tal forma que o braço da alavanca era igual tanto acima como abaixo do referido nível. Com a fixação das extremidades C3 e C7 nas plataformas com acrílico, resultaram livres C4 e C6 para a fixação com placa e parafusos, e C5 para a corpectomia.

A corpectomia central realizada nos modelos foi semelhantes às realizadas na prática cirúrgica ${ }^{(6-8,11,17-20,52-58)}$ sendo retirada a parte central do corpo vertebral, os discos superior e inferior, as paredes anterior e posterior, permanecendo íntegras as paredes laterais. Esta corpectomia foi realizada na área de menos resistência do corpo vertebral - área central - de acordo com Brown et al. ${ }^{(59)}$, permanecendo intactas as regiões laterais, - as mais resistentes do corpo vertebral. Ao contrário, nas fraturas por explosão do corpo vertebral, as paredes laterais também estarão fraturadas. A proposta desta pesquisa foi executar os testes com as paredes laterais íntegras; mesmo assim, em razão de dúvidas em relação a este detalhe, estabeleceu-se um caso piloto para estudar o comportamento de um modelo experimental, em que as paredes laterais estivessem seccionadas.

Neste sentido, a análise desta comparação entre o Grupo Controle, em que as paredes laterais estavam íntegras, e o caso piloto, em que as paredes laterais estavam seccionadas, resultou que, no caso piloto, a falha inicial ocorreu com uma carga bem menor, ressaltando a importância destas paredes íntegras na resistência do conjunto. Em virtude destes resultados, manteve-se o caso piloto com este comportamento isolado, sujeito a crítica, apenas para a discussão, sem participar das conclusões.

Ainda em relação à integridade das paredes laterais, fica clara a ilação de que a corpectomia realizada em caso de estenose do canal, contanto com a integridade das paredes laterais, tornaria o procedimento mais estável do que num caso de fratura por explosão em que também fosse realizada corpectomia e fixação com placa e parafusos.

Estudo radiográfico foi realizado para permitir a observação da não penetração dos parafusos na cortical posterior do corpo vertebral e sua inclinação em relação aos discos intervertebrais. Não houve intenção de fazer análise das radiografias com intuito de interpretar resultados.

Neste estudo foram estabelecidos três tipos de fixação dos conjuntos vertebrais, utilizando três conceitos diferentes de osteossíntese, que englobam a grande maioria das placas que existem no mercado.

A placa tipo $\mathrm{H}$ descrita por Orozco e Llovettapies ${ }^{(8)}$ usa o conceito da dupla fixação proximal e distal, determinando um efeito antirotatório. A placa $1 / 3$ de tubo $A O^{(6,7)}$ é uma placa reta estreita com fixação única proximal e distal. Embora não tenha sido utilizado, tanto a placa1/3 de tubo com a tipo $\mathrm{H}$ tinham disponíveis furos correspondentes ao espaço da corpectomia, onde poderia ser fixado um eventual enxerto. Diferentemente, a placa de Mendonça ${ }^{(20)}$ não possui o furo intermediário, além de ser mais espessa e contar com duas garras proximais e duas distais.

Do ponto de vista de concepção, a placa de Mendonça seria a mais resistente, não apresentando ponto frágil na sua parte média, além de possuir as garras com um pretenso efeito anti-rotatório. Na realidade, esta aparente maior resistência redundou em menor capacidade de absorção de carga, produzindo falha inicial com valores menores ao comparar-se gradualmente com as placas 1/3 de tubo e as tipo $\mathrm{H}$.

A fixação em um único eixo observada nas placas de 1/3 de tubo e de Mendonça, quando associada aos movimentos de flexão e compressão, determinou um efeito rotatório que produziu a ruptura dos corpos vertebrais adjacentes (C4 e C6) à corpectomia, (C5) a partir dos pontos de fixação dos parafusos. Por outro lado, a placa em $\mathrm{H}$, menos espessas e contando com a dupla fixação com parafusos em posição ântero-lateral, absorveram melhor a carga, graças a este seu desenho anti-rotacional. Por esta razão a ruptura óssea nestes grupos de placas ocorreu na média com cargas maiores do que as observadas nos outros dois tipos de placas.

A placa tipo $\mathrm{H}$ acompanhava a deformação sofrida pelo conjunto quando submetida a carga em compressão, o que também foi observado por Ulrich(60)

Assim, com base nos resultados, a carga necessária para provocar a falha inicial e a resistência máxima foi maior no grupo fixado com as placas em $\mathrm{H}$, embora este resultado não seja estatisticamente significante em relação aos obtidos nos outros dois modelos de placas. Ao se compararem os Grupos com placas 1/3 de tubo e de Mendonça ao Grupo Controle, observou-se que as cargas necessárias para produzirem falha inicial e resistência máxima foram estatisticamente menores. Já o Grupo de Placas em H, estatisticamente, comportou-se de forma semelhante ao Grupo Controle, suportando melhor a carga de compressão.

Os resultados sustentam a suposição de que o melhor desempenho biomecânico do Grupo das placas em H, em relação ao Grupo das placas de 1/3 e tubo e ao Grupo das placas de Mendonça deva-se ao seu desenho, uma vez que sua fixação não se dá em um único eixo, como nas outras duas placas. Isto impediria o surgimento precoce do movimento associado de rotação, que 
em última análise provocou o escape das placas 1/3 de tubo e Mendonça com cargas menores.

O melhor desempenho do Grupo Controle em relação aos demais está relacionado, por paradoxal que pareça, à ausência de fixação, pois ficou evidente que a presença do material de síntese provocou efeito rotatório que, acrescido das perfurações dos parafusos - áreas de enfraquecimento ósseo -, produziu a ruptura do conjunto fixado com o escape do material de síntese.

Apesar dos testes não reproduzirem movimentos mais próximos da realidade, isto é, pequenos movimentos repetitivos (forças cíclicas) e sim uma carga única e progressiva, os mesmos trouxeram informações interessantes, tais como o efeito rotatório que surgiu com a instalação das osteossínteses, principalmente as que eram fixadas em um único eixo.

A evolução desta linha de pesquisa certamente acrescentará novos dados a esta discussão, promovendo progressos no conceito de fixação interna da coluna cervical.

\section{REFERÊNCIAS BIBLIOGRÁFICAS}

1. Panjabi MM, Duranceau JS, Oxland TR, Rowen CE. Multidirectional instabilities of traumatic cervical spine injuries in a porcine model. Spine. 1989; 14:1111-31.

2. Roaf R. A study of the mechanics of spinal injuries. J Bone Joint Surg Br. 1960; 42: 810-23.

3. Yoganandan N, Sances A Jr; Pintar F, Maiman D, Reinartz J, Cusick JF et al. Injury biomechanics of the human cervical column. Spine. 1990; 15:1031-9.

4. Panjabi MM, White AA 3rd, Johnson RM. Cervical spine mechanics as a function of transection of components. J Biomech. 1975; 8:327-36.

5. Smith GW, Robinson RA. Anterolateral cervical disk removing and interbody fusion for cervical disk syndrome. Bull Johns Hopkins Hosp. 1955; 96:223-4

6. Bohler G, Gaudernak T. Anterior plate stabilization for fracture dislocations of the lower cervical spine. J Trauma. 1980; 20:203-5.

7. Franco JS. Tratamento da fratura-luxação da coluna cervical (C3-C7). Rev Bras Ortop 1992; 27:131-7.

8. Orozco DR, Llovettapies J. Osteosintesis en las fractures de raquis cervical: nota techinical. Rev Orthop Traumatol. 1970; 14:285-8.

9. Whitehill R, Sirna EC, Young DC, Cantrell RW. Late esophageal perforation from na autogenous bone graft. J Bone Joint Surg Am. 1985; 67:644-5

10. Morscher E, Sutter F, Jenny H, Olerud S. Die vordere verplattung der halswirbesaule mit dem hohlschrauben-plattensystem aus titanium. Chirurg. 1986; 57:702-7.

11. Bohlman $\mathrm{HH}$. Acute fractures and dislocations of the cervical spine. J Bone Joint Surg Am. 1979; 61:1119-42.

12. Cooper PR. Posteriorstabilization of cervical spine fractures and subluxations using plates and screws. Neurosurgery. 1988; 29:300-6.

13. Magarl F, Grob D, Seemann P. Stable dorsal fusion of the cervical spine (C2-T1) using Hook-plates. In: Kehr P, Weidner A. Cervical spine. Berlin: Springer Verlag; 1987. p. 217-21.

14. Rogers WA. Fractures and dislocations of the cervical spine: an end result study. J Bone Joint Surg Am. 1957; 39:341-7.

15. Roy-Camille R, Saillant G, Berteaux D, Serge MA. Early management of spinal injuries. In: Recent Advances in Orthopaedics. Edinburgh: Churchill; 1979. p. 57-87.

16. Stauffer ES. Wiring techniques of the posterior cervical spine for the treatment of trauma Orthopaedics. 1988; 11:1543-8.

17. Barros Filho TEP. Descompressão anterior no tratamento das lesões traumáticas da coluna cervical. Indicações técnicas e resultados [tese]. São Paulo: Faculdade de Medicina da Universidade de São Paulo, 1990.

18. CasparW, Barbier DD, Klara PM. Anterior cervical fusion and Caspar plate stabilization for cervical trauma. Neurosurgery. 1989; 25:491-502.

19. Oliveira JC. Anterior plate fixation of traumatic lesions of the lower cervical spine. Spine. $1987 ; 12: 324-9$

20. Mendonça-Netto ABF. Tratamento das luxações da coluna cervical com lesão medular [dissertação]. São Paulo: Faculdade de Medicina da Universidade de São Paulo; 1981.

21. Machado IR. Estudo experimental do trauma em flexão do segmento médio e inferior da coluna cervical [dissertação]. São Paulo, Escola Paulista de Medicina; 1993.

22. Puertas ED. Consideraçõ̉es sobre o tratamento cirúrgico das instabilidades cervicais por artrodese posterior: com enfoques biomecânicos [tese]. São Paulo: Escola Paulista de Medicina; 1990

23. Rossi JDMBA, Barros Filho TEP, Bollinger Neto R, Leivas TP, Luzo CAM, Novo JRT. Amarria interespinhosa versus amarria sublaminar. Estudo experimental comparativo. Rev Bras Ortop. 1987; 22:79-83.

24. Panjabi MM. Biomechanical evaluation of spinal fixation devices. I.A conceptual framework. Spine. 1988; 13:1129-34

25. Shimano AC, Paulin JBP, Moro CA, Terra O, Pereira LH, Mazzocato FC. Projeto de uma máquina universal com recursos para testes de material biológico. Rev Bras Eng. 1990; 7:391-7.

26. Smith TJ. In vitro spinal biomechanics: experimental methods and apparatus. Spine. 1991; 16:1204-10.

27. Panjabi MM, White AA 3rd. Biomechanics A to Z. In: Clinical biomechanics of the spine. $2^{a}$ ed. Philadelphia: Lippincott; 1990. p.635-96.

28. Coe JD, Warden KE, Sutterlin CE, Mcfee PC. Biomechanical evaluation of cervical spine stabilization methods in a human cadaveric model. Spine. 1989; 14:1122-30.

29. Sutterlin CE, Mcafee PC, Warden KE, Rey RM, Farey ID. A biomechanical evaluation of cervical spine stabilization methods in a bovine model. Static and cyclical loading. Spine. 1988; 13:795-802

30. Allen BL, Ferguson RI, Lehman TR, Brien RP. A mechanistic classification of closed indirect fractures and dislocation of the lower cervical spine. Spine. 1982; 7:1-27.

\section{CONCLUSÕES}

1. As osteossínteses utilizadas não aumentaram a resistência à compressão, quando comparadas ao controle, uma vez que a presença de material de fixação interna diminuiu a estabilidade, fazendo com que a falha inicial ocorresse precocemente, com cargas inferiores ao Grupo Controle.

2. Em relação à falha inicial houve diferenças significativas entre o Grupo Controle e os Grupos com placa de 1/3 de tubo e de Mendonça, porém não houve diferença do Grupo das placas tipo $\mathrm{H}$.

3. Em relação à resistência máxima não houve diferença significativa na comparação entre os Grupos de osteossínteses e o Grupo Controle.

4. A osteossíntese, utilizando placa tipo $\mathrm{H}$, conferiu maior estabilidade quando comparada às outras osteossínteses, embora as diferenças não sejam significativas.

31. Danis F. Spinal instability as defined by the three column spine concept in acute spina trauma. Clin Orthop Relat Res. 1984; (189):65-76.

32. Eurell JAC, Kazarian LE. The scanning electron microscopy of compressed vertebral bodies. Spine. 1982; 7:123-8

33. Holdsworth FW. Fractures dislocations and fracture-dislocations of the spine. $J$ Bone Joint Surg Br. 1963; 45:6-20

34. Panjabi MM, Hausteld JN, White AA 3rd. A biomechanical study of the ligaments stability of the thoracic spine mann. Acta Orthop Scand. 1981; 52: 315-26.

35. Davis AG. Tensile strength of the anterior longitudinal ligament in relation to treatment of 132 crush fractures of the spine. J Bone Joint Surg Br. 1938; 20: 429-38.

36. Miller JAA, Schultz AB, Warwick DN, Spencer DL. Mechanical prospect of lumbar spine motion segments under large loads. J Biomech. 1986; 19:79-84

37. Sedlin ED, Hirsch C. Factors affecting the determination of the physical properties of femural cortical bones. Acta Orthop Scand. 1966; 37: 29-48.

38. Tkaczuk H. Tensil properties of human lumbar longitudinal ligaments. Acta Orthop Scand. 1968; 115(Suppl):117-21.

39. Atkinson PJ. Variation in trabecular structure of vertebral with age. Calcif $T$ issue Res. 1967; 1:24-32.

40. Beatson TR. Fractures and dislocations of the cervical spine. J Bone Joint Surg Br. 1963; 45:21-35.

41. Gosch HH, Gooding E, Schneider RC. Na experimental study of cervical spine and crd injurie. J Trauma. 1972; 12:570-5.

42. Johnson RM, Crelin ES, White AA 3rd, Panjabi MM, SouthwickWO. Some new observation of the functional anatomy of lower cervical spine. Clin Orthop Relat Res. 1975; (111): 192-200

43. Nova Monteiro JA, Giesta C. Fraturas e luxações da coluna cervical. Rev Med Rio de Janeiro. 1970; 37: 345-57.

44. Fielding JW, Burstein AA, Frankel VH. The nuchal ligament. Spine. 1976; 1:3-14

45. Nolan JP Jr, Sherk Hh. Biomechanical evaluation of the extensor musculature of the cervical spine. Spine.1988;13:9-11.

46. Halliday DR, Sullivan CR, Hollinshead WH, Bahn RC. Torn cervical ligaments: necropsy examination of the normal cervical region of the spinal column. J Trauma. 1964; 4:219-32.

47. WHITE AA 3rd, Johnson RM, Panjabi MM Southwick WO. Biomechanical analysis of clinical stability in the cervical spine. Clin Orthop Relat Res. 1975; (109):85-96.

48. Panjabi MM, Goel VR, Takada K. Physiologic strains in the lumbar spinal ligaments. Spine.1982; 7:192-203

49. Barros Filho TEP, Taricco MA, Oliveira RP, Greve JAM, Santos LCR, Napoli MMM. Estudo epidemiológico dos pacientes com traumatismos da coluna vertebral e déficit neurológico, internados no Instituto de Ortopedia e Traumatologia do Hospital das Clínicas da Faculdade de Medicina da Universidade de São Paulo. Rev Hosp Clin São Paulo. 1990; 45:123-6.

50. Franco JS, Silva DH, Solino JL, Amaral NCP. Avaliação neurológica das lesões traumáticas da coluna cervical. Rev Bras Ortop. 1989; 24:23-7.

51. Solino JL, Melo MFFV, Silva DHA, Elias N. Traumatismos da coluna vertebral. Avaliação

de etiologia, incidência e frequência. Rev Bras Ortop. 1990; 25: 185-90.
52. Boni M. Denaro V. Surgical treatment of traumatic lesions of the middle and lower cervical spine (C3-C7). Ital J Orthop Traumatol. 1980; 6:305-20.

53. Cabanella ME, Ebersold MJ. Anterior plate estabilization for burst tear drop fracture of the Cabanella ME, Ebersold MJ. Anterior plate
cervical spine. Spine. 1988; 13:888-91.

54. Herrmann HD. Metal plate fixation after anterior fusion of unstable fracture dislocation of the cervical spine. Acta Neurochir. 1975; 32:101-11.

55. McAfee PC, Bohlman HH, Riley LH Jr, Robinson RA, Southwick WO, Nachlas NE. One stage anterior cervical decompression and posterior with circunferential arthrodesis. $J$ Bone Joint Surg Am. 1989; 71: 78-88

56. Seemann P, Magerl F, Grob D. Anterior interbody fusion with transdical decompression in the cervical spine. In: Kehr P, Weidner A. Cervical spine. Berlim: Springer Verlag, 1987. p. 273-7.

57. Senegas J, Gauzers JM. Plaidoyer por la chirurgie anterieure dans le traitement des traumatismes graves des cinq dernieres vertebres cervicales. Rev Chir Orthop. 1976; 62:123-8

58. Whitehill R, Reger SI, Kett RL, Payne R, Barry J. Reconstruction of the cervical spine following anterior vertebral body resection: a mechanical analysis of a canine experimental model. Spine. 1984; 9:240-5

59. Brown JA, Havel P, Ebraheim N, Greenblatt SH, Jackson WT. Cervical stabilization by plate and bone fusion. Spine. 1988; 13:236-40.

60. Ulrich C, Worsdorfer O, Claes L, Magerl F. Comparative study of stability of anterior and posterior cervical spine fixation procedures. Arch Orthop Trauma Surg. 1987; 106:226-31. 